Article

\title{
The Distribution Function of a Probability Measure on a Linearly Ordered Topological Space
}

\author{
José Fulgencio Gálvez-Rodríguez *,` and Miguel Ángel Sánchez-Granero † ${ }^{\text {† }}$ \\ Departamento de Matemáticas, Universidad de Almería, 04120 Almería, Spain; misanche@ual.es \\ * Correspondence: jgr409@ual.es; Tel.: +34-950-015-005 \\ + These authors contributed equally to this work.
}

Received: 30 July 2019; Accepted: 11 September 2019; Published: 18 September 2019

Abstract: In this paper, we describe a theory of a cumulative distribution function on a space with an order from a probability measure defined in this space. This distribution function plays a similar role to that played in the classical case. Moreover, we define its pseudo-inverse and study its properties. Those properties will allow us to generate samples of a distribution and give us the chance to calculate integrals with respect to the related probability measure.

Keywords: probability; measure; $\sigma$-algebra; Borel $\sigma$-algebra; distribution function; cumulative distribution function; sample; linearly ordered topological space

\section{Introduction}

This work collects some results on a theory of a cumulative distribution function on a linearly ordered topological space (LOTS).

Moreover, we show that this function plays a similar role to the one it plays in the classical case and study its pseudo-inverse, which allows us to generate samples of the probability measure that we use to define the distribution function.

The main goal of this paper is to provide a theory of a cumulative distribution function on a space with a linear order. Furthermore, we show that a cumulative distribution function in this context plays a similar role to that played by a distribution function in the classical case. Recall that in the classical case, the cumulative distribution function (in short, cdf) of a real-valued random variable $X$ is the function defined by $F_{X}(x)=P[X \leq x]$ and it satisfies the following properties:

1. $\quad F$ is non-decreasing, which means that for each $x, y \in \mathbb{R}$ with $x<y$, we have $F(x) \leq F(y)$.

2. $F$ is right-continuous, which means that $F(a)=\lim _{x \rightarrow a^{+}} F(x)$, for each $a \in \mathbb{R}$. Furthermore, $\lim _{x \rightarrow-\infty} F(x)=0$ and $\lim _{x \rightarrow+\infty} F(x)=1$.

Moreover, given a cdf in an ordered space, we define its pseudo-inverse and study its properties. In the classical case, when a cdf, $F$, is strictly increasing and continuous it holds that $F^{-1}(p)$ is the unique real number $x$, such that $F(x)=p$, for each $p \in[0,1]$. In that case, this defines the inverse of the distribution function.

The inverse is not unique for some distributions (for example in the case where the density function $f_{X}(x)=0$, for each $a<x<b$, causing $F_{X}$ to be constant). This problem can be solved by defining, for each $p \in[0,1]$, the pseudo-inverse of the distribution function by $F^{-1}(p)=\inf \{x \in \mathbb{R}$ : $F(x) \geq p\}$.

The inverse of a cdf let us generate samples of a distribution. Indeed, let $X$ be a random variable whose distribution can be described by the cdf $F$. Our goal is to generate values of $X$ according to this distribution. The inverse transform sampling method is as follows: generate a random number, $u$, from the standard uniform distribution in the interval $[0,1]$ and then consider $x=F^{-1}(u)$. 
Roughly speaking, given a continuous uniform random variable, $U$, in $[0,1]$ and a cdf, $F$, the random variable $X=F^{-1}(U)$ has distribution $F$ (or, $X$ is distributed $F$ ).

For further reference about the pseudo-inverse of $F$ see, for example, [1], Chapter 1.

In [2] (see also [3-5]), it is proved the equivalence between probability measures and fuzzy intervals in $\mathbb{R}$. Probabilistic metric and normed spaces, which were introduced in [6,7] respectively, provide a different (more studied) relation between topology and probability measures. For further reference about this topic see, for example, [8-10].

When we work with probability measures on $\mathbb{R}$, the use of a cdf to fully describe the probability measure is quite handy: it is easier to describe (or define) a cdf than a probability measure, its pseudo-inverse can be used to generate samples and it determines the probability measure. Based on this, it seems interesting to extend the theory of a cdf to a more general setting. Since the main properties of a cdf are related with order and continuity, a linearly ordered topological space seems to be the natural place where such a theory could be developed. The main goal of this paper is to provide a first study of the definition and properties of a cdf in a separable LOTS. Another way to generalize the concept of distribution function can be found in [11].

Our study begins with the proof of several properties of the order topology in a separable LOTS by taking advantage of a certain type of sequences in some cases (see Section 2). In Section 3, we define the cumulative distribution function (cdf) of a probability measure on a separable LOTS and prove that its properties are quite similar to those which are well-known in the classical case, described in the previous paragraphs. Furthermore, from a cdf $F$, we will define $F_{-}$which plays a similar role to that played by $\lim _{x \rightarrow a^{-}} F(x)$ in the classic theory of distribution functions. We also use $F$ and $F_{-}$to get the measure of an interval. On the other hand, Section 4 is dedicated to proving some aspects related to the discontinuities of a cdf. Section 5 introduces the concept of pseudo-inverse for a cdf defined on a separable LOTS, which is a measurable function. Finally, Section 6 shows that by using the pseudo-inverse of a cdf, it is possible to calculate integrals with respect to the initial probability measure and generate samples of a distribution in case that the separable LOTS is compact.

\section{Preliminaries}

\subsection{Measure Theory}

Now we recall some definitions related to measure theory from [12]. Let $X$ be a set, then there are several classes of sets of $X$. If $\mathcal{R}$ is a non-empty collection of subsets of $X$, we say that $\mathcal{R}$ is a ring if it is closed under complement and finite union. Furthermore, given $\mathcal{Q}$ is a non-empty collection of subsets of $X$ it is said to be an algebra if it is a ring such that $X \in \mathcal{Q}$. Moreover, a non-empty collection of subsets of $X, \mathcal{A}$, is a $\sigma$-algebra if it is closed under complement and countable union and $X \in \mathcal{A}$.

For a given topological space, $(X, \tau), \mathcal{B}=\sigma(\tau)$ is the Borel $\sigma$-algebra of the space, i.e., it is the $\sigma$-algebra generated by the open sets of $X$.

Definition 1 ([12], Section 7). Given a measurable space $(\Omega, \mathcal{A})$, a measure $\mu$ is a non-negative and $\sigma$-additive set mapping defined on $\mathcal{A}$ such that $\mu(\varnothing)=0$.

A set mapping is said to be $\sigma$-additive if $\mu\left(\bigcup_{n=1}^{\infty} A_{n}\right)=\sum_{n=1}^{\infty} \mu\left(A_{n}\right)$ for each countable collection $\left\{A_{n}\right\}_{n=1}^{\infty}$ of pairwise disjoint sets in $\mathcal{A}$.

Each measure is monotonous, which means that $\mu(A) \leq \mu(B)$, for each $A \subseteq B$. Moreover, it is continuous in the next sense: if $A_{n} \rightarrow A$, then $\mu\left(A_{n}\right) \rightarrow \mu(A)$. Furthermore, if $A_{n}$ is a monotonically non-decreasing sequence of sets (which means that $A_{n} \subseteq A_{n+1}$, for each $n \in \mathbb{N}$ ) then $\mu\left(A_{n}\right) \rightarrow$ $\mu\left(\bigcup_{n \in \mathbb{N}} A_{n}\right)$. If $A_{n}$ is monotonically non-increasing (which means that $A_{n+1} \subseteq A_{n}$, for each $n \in \mathbb{N}$ ), then $\mu\left(A_{n}\right) \rightarrow \mu\left(\bigcap_{n \in \mathbb{N}} A_{n}\right)$. 


\subsection{Ordered Sets}

First we recall the definition of a linear order and a linearly ordered topological space:

Definition 2 ([13], Chapter 1). A partially ordered set $(P, \leq)$ (that is, a set $P$ with the binary relation $\leq$ that is reflexive, antisymmetric and transitive) is totally ordered if every $x, y \in P$ are comparable, i.e., $x \leq y$ or $y \leq x$. In this case, the order is said to be total or linear.

For further reference about partially ordered sets see, for example, [14].

Definition 3 ([15], Section 1). A linearly ordered topological space (abbreviated LOTS) is a triple $(X, \tau, \leq)$ where $(X, \leq)$ is a linearly ordered set and where $\tau$ is the topology of the order $\leq$.

The definition of the order topology is the following one:

Definition 4 ([16], Part II, 39). Let $X$ be a set which is linearly ordered by $<$, we define the order topology $\tau$ on $X$ by taking the sub-basis $\{\{x \in X: x<a\}: a \in X\} \cup\{\{x \in X: x>a\}: a \in X\}$.

From a linear order, $\leq$, in $X$ we define

Definition 5. Let $a, b \in X$ with $a \leq b$, we define the set $] a, b]=\{x \in X: a<x \leq b\}$. Analogously, we define $] a, b[,[a, b]$ and $[a, b[$. Moreover, $(\leq a)$ is given by $(\leq a)=\{x \in X: x \leq a\} .(<a),(\geq a)$ and $(>a)$ are defined similarly.

Notation 1. Let $a \in X$, we will also use $] a, \infty[$ and $[a, \infty[$ to denote $(>a)$ and $(\geq a)$, respectively. Similarly, ]$-\infty, a[$ and $]-\infty, a]$ will also denote $(<a)$ and $\leq a$, respectively.

Remark 1. Please note that an open basis of $X$ with respect to $\tau$ is given by \{]$a, b[: a<b, a, b \in(X \cup$ $\{-\infty, \infty\})\}$.

For our study we need to introduce some terminology.

Definition 6 ([15], Section 1$)$. Let $(X, \leq)$ be a linearly ordered set. A subset $C \subseteq X$ is said to be convex in $X$ if, whenever $a, b \in C$ with $a \leq b$, then $\{x \in X: a \leq x \leq b\}$ is a subset of $C$.

Proposition 1 ([16], Part II, 39). Any subset $A \subseteq X$ can be uniquely expressed as a union of disjoint, non-empty, maximal convex sets in $A$, called convex components.

The definition of interval is the following one:

Definition 7 ([15], Section 1). An interval of $X$ is a convex subset of $X$ with two endpoints in $X$, which can belong to the interval or not.

For convention, we will assume that $\infty$ and $-\infty$ can be the endpoints of intervals.

Definition 8 ([17], Defs 2.16, 2.17). Let $P$ be an ordered set and let $A \subseteq P$. Then:

1. I is called a lower bound of $A$ if, and only if we have $l \leq a$, for each $a \in A$.

2. $u$ is called an upper bound of $A$ if, and only if we have $u \geq a$, for each $a \in A$.

Definition 9. Given $A \subseteq X$, we denote by $A^{l}$ and $A^{u}$, respectively, the set of lower and upper bounds of $A$.

Definition 10 ([17], Def 3.18). Let $P$ be an ordered set and let $A \subseteq P$. Then: 
1. The point $u$ is called the lowest upper bound or supremum or join of $A$ iff $u$ is the minimum of the set $A^{u}$.

2. The point $u$ is called the greatest lower bound or infimum or meet of $A$ iff $l$ is the maximum of the set $A^{l}$.

Proposition 2 ([16], Part II, 39). The order topology on X is compact if, and only if the order is complete, i.e., if, and only if, every non-empty subset of $X$ has a greatest lower bound and a least upper bound.

Remark 2. In the rest of the paper, unless otherwise stated, X will be a separable LOTS and a measure in $X$ will be with respect to the Borel $\sigma$-algebra of $X$.

\section{The order in $X$}

In this section, we study some properties (mainly topological) of a separable LOTS.

The definition of the topology $\tau$ suggest the next

Definition 11. Let $x \in X$, it is said to be a left-isolated (respectively right-isolated) point if $(<x)=\varnothing$ (respectively $(>x)=\varnothing$ ) or there exists $z \in X$ such that $] z, x[=\varnothing$ (respectively there exists $z \in X$ such that ]$x, z[=\varnothing)$. Moreover, we will say that $x \in X$ is isolated if it is both right and left-isolated.

Lemma 1. Let $A, B \subseteq X$ be such that $A^{l}=B^{l}$ (respectively $A^{u}=B^{u}$ ). If there exists inf $A$ (respectively sup $A)$, then there exists $\inf B($ respectively sup $B)$ and $\inf A=\inf B($ respectively $\sup A=\sup B$ ).

Proof. Let $A, B \subseteq X$ be such that $A^{l}=B^{l}$ and suppose that there exists $\inf A$. It holds that $x \leq \inf A$, for each $x \in A^{l}$. Now, since $A^{l}=B^{l}$, we have that $\inf A \in A^{l}=B^{l}$ and $x \leq \inf A$, for each $x \in B^{l}$, i.e., $\inf A=\inf B$.

The case in which $A^{u}=B^{u}$ and there exists sup $A$ can be proven analogously.

Proposition 3. Let $A \subseteq X$ be a non-empty subset such that it does not have a minimum (respectively a maximum), then there exists a sequence $a_{n} \in A$ such that $a_{n+1}<a_{n}$, for each $n \in \mathbb{N}$ and $A^{l}=\left\{a_{n}: n \in \mathbb{N}\right\}^{l}$ (respectively $a_{n+1}>a_{n}$, for each $n \in \mathbb{N}$ and $A^{u}=\left\{a_{n}: n \in \mathbb{N}\right\}^{u}$ ).

Proof. Let $D$ be a dense and countable subset of $X$ and consider $D_{A}=\left\{d \in D: d \notin A^{l}\right\}$. Please note that the fact that $d \notin A^{l}$ is equivalent to the existence of $a \in A$ such that $a<d$. Moreover, $D_{A} \subseteq D$, so $D_{A}$ is countable, so we can enumerate it as $D_{A}=\left\{d_{n}: n \in \mathbb{N}\right\}$. Given $d_{1} \in D_{A}$, there exists $a_{1} \in A$ such that $a_{1}<d_{1}$. Suppose that $a_{n} \in A$ is a sequence defined by $a_{n}<d_{n}$ and $a_{n}<a_{n-1}$, for each $n \in \mathbb{N}$. We define $a_{n+1}$ as follows. Since there does not exists the minimum of $A$, we can choose $a \in A$ such that $a<a_{n}$. Apart from that, there exists $a^{\prime} \in A$ such that $a^{\prime}<d_{n+1}$. Hence, if we consider $a_{n+1}=\min \left\{a, a^{\prime}\right\}$, then $a_{n+1}<a_{n}$ and $a_{n+1}<d_{n+1}$. Recursively, we have defined a sequence $a_{n} \in A$ such that $a_{n+1}<a_{n}$ and $a_{n}<d_{n}$, for each $n \in \mathbb{N}$.

Now we prove that $A^{l}=\left\{a_{n}: n \in \mathbb{N}\right\}^{l}$.

$\subseteq)$ This is obvious.

) Let $x \in X$ be such that $x \leq a_{n}$, for each $n \in \mathbb{N}$. Now we prove that $x \leq a$, for each $a \in A$. For that purpose, let $a \in A$. Since there does not exist the minimum of $A$, there exist $a^{\prime} \in A$ such that $a^{\prime}<a$ and $a^{\prime \prime} \in A$ such that $a^{\prime \prime}<a^{\prime}$. Consequently, $] a^{\prime \prime}, a[$ is a non-empty open set in $X$ with respect to $\tau$, so we can choose $d \in D \cap] a^{\prime \prime}, a\left[\right.$. Hence, $d>a^{\prime \prime}$, which implies that $d \in D_{A}$. It follows that there exists $n_{0} \in \mathbb{N}$ such that $d=d_{n_{0}}$. Therefore, $x \leq a_{n_{0}}<d_{n_{0}}<a$, which lets us conclude that $x \leq a$.

Convex subsets can be described as countable union of intervals.

Corollary 1. Let $A \subseteq X$ be a convex subset. Then it holds that:

1. If there exist both minimum and maximum of $A$, then $A=[\min A, \max A]$.

2. If there does not exist the minimum of $A$ but it does its maximum, then there exists a decreasing sequence $a_{n} \in A$ such that $\left.\left.A=\bigcup_{n \in \mathbb{N}}\right] a_{n}, \max A\right]$. 
3. If there does not exist the maximum of $A$ but it does its minimum, then there exists an increasing sequence $b_{n} \in A$ such that $A=\bigcup_{n \in \mathbb{N}}\left[\min A, b_{n}[\right.$.

4. If there does not exist the minimum of $A$ nor its maximum, then there exist a decreasing sequence $a_{n} \in A$ and an increasing one $b_{n} \in A$ such that $\left.A=\bigcup_{n \in \mathbb{N}}\right] a_{n}, b_{n}[$.

\section{Proof.}

1. It is clear.

2. Since $A$ is non-empty and there does not exist the minimum of $A$, by Proposition 3 , we can choose a sequence $a_{n} \in A$ such that $a_{n+1}<a_{n}$, for each $n \in \mathbb{N}$ and $A^{l}=\left\{a_{n}: n \in \mathbb{N}\right\}^{l}$. Now we prove that $\left.\left.A=\bigcup_{n \in \mathbb{N}}\right] a_{n}, \max A\right]$.

$\subseteq)$ Let $x \in A$. Since $A$ does not have a minimum, then $x \notin A^{l}$ which implies that $x \notin\left\{a_{n}: n \in\right.$ $\mathbb{N}\}^{l}$. Then there exists $n \in \mathbb{N}$ such that $a_{n}<x$. Consequently, $\left.\left.x \in \bigcup_{n \in \mathbb{N}}\right] a_{n}, \max A\right]$.

?) Let $\left.\left.x \in \bigcup_{n \in \mathbb{N}}\right] a_{n}, \max A\right]$, then there exists $n \in \mathbb{N}$, such that $a_{n}<x \leq \max A$. Hence, the fact that $A$ is convex together with the fact that $a_{n} \in A$ give us that $x \in A$.

3. It can be proven similarly to the previous item.

4. Since $A$ is non-empty and there does not exist the minimum of $A$ nor its maximum, by Proposition 3, we can choose two sequences $a_{n}, b_{n} \in A$ such that $a_{n+1}<a_{n}$ and $b_{n+1}>b_{n}$, for each $n \in \mathbb{N}$ and $A^{l}=\left\{a_{n}: n \in \mathbb{N}\right\}^{l}, B^{u}=\left\{b_{n}: n \in \mathbb{N}\right\}^{u}$. Now we prove that $\left.A=\bigcup_{n \in \mathbb{N}}\right] a_{n}, b_{n}[$.

$\subseteq$ ) Let $x \in A$. Since $A$ does not have a minimum nor a maximum then $x \notin A^{l}$ and $x \notin A^{u}$, which implies that $x \notin\left\{a_{n}: n \in \mathbb{N}^{l}\right.$ and $x \notin\left\{b_{n}: n \in \mathbb{N}\right\}^{u}$, then there exists $n_{1} \in \mathbb{N}$ and $n_{2} \in \mathbb{N}$ such that $a_{n_{1}}<x<b_{n_{2}}$. If we define $n=\max \left\{n_{1}, n_{2}\right\}$, then it holds that $a_{n}<x<b_{n}$ and we conclude that $\left.x \in \bigcup_{n \in \mathbb{N}}\right] a_{n}, b_{n}[$.

) Let $\left.x \in \bigcup_{n \in \mathbb{N}}\right] a_{n}, b_{n}\left[\right.$, then there exists $n \in \mathbb{N}$, such that $a_{n}<x<b_{n}$. Hence, the fact that $A$ is convex together with the fact that $a_{n}, b_{n} \in A$ give us that $x \in A$.

Similarly, convex open subsets can be described as countable union of open intervals.

Corollary 2. Let $A$ be an open and convex subset of $X$, then $A$ is the countable union of open intervals.

Proof. We distinguish some cases depending on whether there exist the maximum or the minimum of $A$ :

1. Suppose that there does not exist the maximum of $A$ nor its minimum, then by Corollary 1 , it holds that $A$ can be written as the countable union of open intervals.

2. Suppose that there does not exist the minimum of $A$ but it does its maximum. By the previous corollary, it holds that $\left.\left.A=\bigcup_{n \in \mathbb{N}}\right] a_{n}, \max A\right]$. Now note that the fact that $A$ is open means that $\max A$ is right-isolated so we can write $\left.A=\bigcup_{n \in \mathbb{N}}\right] a_{n}, b[$, where $b$ is the following point to max $A$. Hence, $A$ is the countable union of open intervals.

3. If there exists the minimum of $A$ but not its maximum, we can proceed analogously to claim that $\left.A=\bigcup_{n \in \mathbb{N}}\right] a, b_{n}\left[\right.$ where $a$ is the previous point to $\min A$ and $b_{n}$ is an increasing sequence in $A$.

4. If there exists both minimum and maximum of $A$, then $A=] a, b[$ where $a$ is the previous point to $\min A$ and $b$ is the following one to $\max A$.

Next, we prove that a separable LOTS is first countable.

Proposition 4. $\tau$ is first countable.

Proof. Since $X$ is separable with respect to the topology $\tau$, there exists a countable dense subset $D$ of $X$. Now we prove that given $x \in X$, each of the countable families 
- $\quad \mathcal{B}_{x}=\{\{x\}\}$ if $x$ is isolated.

- $\quad \mathcal{B}_{x}=\{] a, b[: a<x<b, a, b \in D\}$, if $x$ is not left-isolated nor right-isolated.

- $\quad \mathcal{B}_{x}=\{[x, b[: x<b, b \in D\}$ if $x$ is left-isolated but it is not right-isolated.

- $\left.\left.\quad \mathcal{B}_{x}=\{] a, x\right]: a<x, a \in D\right\}$ if $x$ is right-isolated but it is not left-isolated.

is a countable neighborhood basis of $x$ with respect to the topology $\tau$. For that purpose, we prove the next two items:

- Each element of $\mathcal{B}_{x}$ is a neighborhood of $x$, for each $x \in X$. This is clear if we take into account that each element in $\mathcal{B}_{x}$ is an open set with respect to the topology $\tau$ (see Remark 1). Indeed, if $x$ is left-isolated then, given $B \in \mathcal{B}_{x}$, we can write $B=[x, b[$, for some $b \in D$ with $b>x$. Equivalently, $B=] a, b[$, where $a$ is the previous point to $x$ according to the order. The other cases are similar.

- For each neighborhood of $x, U$, there exists $B \in \mathcal{B}_{x}$ such that $B \subseteq U$. Indeed, let $U$ be a neighborhood of $x$, then there exists an open set $G$ such that $x \in G \subseteq U$. Since $G$ is open and $\mathcal{B}=\{] a, b[: a<b\}$ is an open basis, we can consider $a, b$ such that $] a, b[\subseteq G$ and $a<x<b$. Now we distinguish some cases depending on whether $x$ is isolated or not:

- $\quad$ Suppose that $x$ is isolated, then there exist $y, z \in X$ such that $y<x<z$ and $] y, z[=\{x\}$. In this case $\{x\}$ is an element of $\mathcal{B}_{x}$ which is contained in $U$.

- $\quad$ Suppose that $x$ is not left-isolated nor right-isolated. Since $] a, x[$ and $] x, b[$ are both open in $\tau$ and $D$ is dense in $\tau$, we can choose $c \in] a, x[\cap D$ and $d \in] x, b[\cap D$. Furthermore, it holds that $x \in] c, d[\subseteq] a, b[\subseteq G \subseteq U$, which finishes the proof.

- $\quad$ Suppose that $x$ is left-isolated but it is not right-isolated. Then there exists $y \in X$ such that ]$y, x[=\varnothing$ and $] x, z[\neq \varnothing$ for each $z>x$. Since $] x, b[$ is open in $\tau$ and $D$ is dense in $\tau$, we can choose $d \in] x, b[\cap D$. Furthermore, it holds that $x \in[x, d[\subseteq] a, b[\subseteq G \subseteq U$.

- $\quad$ Suppose that $x$ is not left-isolated but it is right-isolated. Then there exists $z \in X$ such that ]$y, x[\neq \varnothing$ and $] x, z[=\varnothing$, for each $y<x$. Since $] a, x[$ is a neighborhood in $\tau$ and $D$ is dense in $\tau$, we can choose $c \in] a, x[\cap D$. Furthermore, it holds that $x \in] c, x] \subseteq] a, b[\subseteq G \subseteq U$.

We can choose a countable neighborhood basis of each point such that its elements are ordered, as next remark shows:

Remark 3. Let $x \in X$, then there exists a countable neighborhood basis of $x, \mathcal{B}_{x}^{\prime}=\{] a_{n}^{\prime}, b_{n}^{\prime}\left[: a_{n}^{\prime}<x<b_{n}^{\prime} ; n \in \mathbb{N}\right\}$ such that $a_{n}$ is a non-decreasing sequence and $b_{n}$ a non-increasing one.

Proof. Indeed, since $\tau$ is first countable, there exists a countable basis of each point. According to the previous proposition, in case that $x$ is not left-isolated nor right-isolated, we have that $\mathcal{B}_{x}=\{] a_{1}, b_{1}[$ : $\left.a_{1}<x<b_{1} ; a_{1}, b_{1} \in D\right\}$ is a countable basis of $x$. Since $D$ is a dense subset in $\tau$ and $] x, b_{1}[$ and $] a_{1}, x[$ are non-empty open sets in $\tau$, there exists $\left.d_{a_{1}} \in D \cap\right] a_{1}, x\left[\right.$ and $\left.d_{b_{1}} \in\right] x, b_{1}\left[\cap D\right.$. Now define $a_{2}=d_{a_{1}}$ and $b_{2}=d_{b_{1}}$. Moreover, there exists $\left.d_{a_{2}} \in D \cap\right] a_{2}, x\left[\right.$ and $\left.d_{b_{2}} \in D \cap\right] x, b_{2}\left[\right.$. Now we define $a_{3}=d_{a_{2}}$ and $b_{3}=d_{b_{2}}$. Recursively we have that $\mathcal{B}_{x}^{\prime}=\{] a_{n}, b_{n}\left[: a_{n}<x<b_{n} ; n \in \mathbb{N}, a_{n}, b_{n} \in D\right\}$ where $a_{n}=d_{a_{n-1}}$ and $b_{n}=d_{b_{n-1}}$. It is clear that $\mathcal{B}_{x}$ is a neighborhood basis of $x$. Moreover, given $n \in \mathbb{N}$ it holds that ]$a_{n+1}, b_{n+1}[\subseteq] a_{n}, b_{n}$ [ by the definition of $a_{n}^{\prime}$ and $b_{n}^{\prime}$. We can proceed analogously to get basis for the right-isolated or left-isolated points. Moreover, note that if $x$ is isolated, the basis given in the previous proposition satisfies the condition given in this remark.

There exists an equivalence between the property of second countable for $\tau$ and the countability of the set of isolated points.

Proposition 5. Let $X$ be a LOTS. $X$ is second countable with respect to the topology $\tau$ if, and only if $X$ is separable and the set of right-isolated or left-isolated points is countable. 
Proof. Let us define $C_{1}$ and $C_{2}$ to be, respectively, the set of left-isolated points and the set of right-isolated points.

$\Leftarrow)$ Let $D$ be a countable dense subset of $X$. Moreover, suppose that $C_{1}$ and $C_{2}$ are countable subsets. Consider the family $\left.\mathcal{B}=\left\{\{x\}: x \in C_{1} \cap C_{2}\right\} \cup\{] a, x\right]: a<x, x \in C_{2}, a \in$ $D\} \cup\left\{\left[x, b\left[: x<b, x \in C_{1}, b \in D\right\} \cup\{] a, b[: a<b, a, b \in D\}\right.\right.$ and note that it is an open basis of $X$ with respect to $\tau$. Furthermore, the countability of the set of right-isolated and left-isolated points gives us that $\mathcal{B}$ is countable. Hence $\tau$ is second countable.

$\Rightarrow$ ) Suppose that $X$ is second countable with respect to $\tau$, then there exists a countable open basis, $\mathcal{B}=\left\{U_{n}: n \in \mathbb{N}\right\}$. Since second countable spaces are separable, we only must prove that $C_{1}$ and $C_{2}$ are countable subsets, which gives us that $C_{1} \cup C_{2}$ is also countable.

- $\quad C_{1}$ is countable: let $x \in C_{1}$ and $b_{1}>x$ with $b_{1} \in D$. Since $\mathcal{B}$ is an open basis and $\left[x, b_{1}\right.$ [ is an open set containing $x$, there exists $n_{x} \in \mathbb{N}$ such that $x \in U_{n_{x}} \subseteq\left[x, b_{1}\left[\right.\right.$. Now let $y \in C_{1}$ with $y \neq x$ and $b_{2} \in D$ with $y<b_{2}$, then there exists $n_{y} \in \mathbb{N}$ such that $y \in U_{n_{y}} \subseteq\left[y, b_{2}\left[\right.\right.$ for $b_{2}>y$. Consequently, $f: C_{1} \rightarrow \mathbb{N}$ given by $f(x)=n_{x}$ is an injective function, which proves the countability of $C_{1}$.

- The countability of $C_{2}$ can be proved similarly to the countability of $C_{1}$.

Now we define the concept of right convergent and left convergent sequence.

Definition 12. Let $x \in X$ and $v$ be a topology defined on $X$. We say that a sequence $x_{n} \in X$ is right $v$-convergent (respectively left $v$-convergent) to $x$ if $x_{n} \stackrel{v}{\rightarrow} x$ and $x_{n} \geq x$ (respectively $\left.x_{n} \leq x\right)$, for each $n \in \mathbb{N}$.

Now we define the concept of monotonically right convergent and monotonically left convergent sequence.

Definition 13. Let $x \in X$ and $v$ be a topology defined on $X$. We say that a sequence $x_{n} \in X$ is monotonically right v-convergent (respectively monotonically left v-convergent) to $x$ if $x_{n} \stackrel{v}{\rightarrow} x$ and $x<x_{n+1}<x_{n}$ (respectively $x_{n}<x_{n+1}<x$ ), for each $n \in \mathbb{N}$.

Proposition 6. Let $x \in X$. Then $x$ is not left-isolated (respectively right-isolated) if, and only if there exists a monotonically left $\tau$-convergent (respectively monotonically right $\tau$-convergent) to $x$ sequence.

Proof. $\Rightarrow$ ) Let $x$ be a non-left-isolated point, then $x \neq \min X$. Since $\tau$ is first countable (by Proposition 4 ), we can consider a countable neighborhood basis of $x, \mathcal{B}_{x}=\{] a_{n}, b_{n}[: n \in \mathbb{N}\}$. Now let $a, b \in X$ be such that $a<x<b$, then there exists $n_{1} \in \mathbb{N}$ such that $a \leq a_{n_{1}}<x$ due to the fact that $\mathcal{B}_{x}$ is a neighborhood basis of $x$. Since $x$ is not left-isolated, we can choose $\left.z_{1} \in\right] a_{n_{1}}, x$. Now we can consider $n_{2} \in \mathbb{N}$ such that $z_{1} \leq a_{n_{2}}<x$ due to the fact that $\mathcal{B}_{x}$ is a neighborhood basis of $x$. Recursively, we can construct a subsequence of $a_{n}, a_{\sigma(n)}$, such that $a_{\sigma(n)}<a_{\sigma(n+1)}<x$ and $a_{\sigma(n)} \rightarrow x$, i.e., $a_{\sigma(n)}$ is a monotonically left $\tau$-convergent sequence to $x$.

The proof is analogous in case that $x$ is not right-isolated.

$\Leftrightarrow)$ Let $x \in X$ and suppose that it is a left-isolated point. If $x=\min X$ the proof is easy. Suppose that $x \neq \min X$, then there exists $z \in X$ such that $] z, x[=\varnothing$. Suppose that there exists a monotonically left $\tau$-convergent sequence to $x, x_{n}$, then it holds that there exists $n_{0} \in \mathbb{N}$ such that $x_{n}>z$, for each $n \geq n_{0}$. Moreover, since $x_{n}<x$, we have that $\left.x_{n} \in\right] z, x[=\varnothing$, which is a contradiction. Hence, $x$ is not left-isolated.

The case in which there exists a monotonically right $\tau$-convergent sequence to $x$ can be proven analogously.

\section{Lemma 2.}

1. If $a_{n}$ is a monotonically left $\tau$-convergent sequence to $a$, then $\cup\left(<a_{n}\right)=(<a)=\cup\left(\leq a_{n}\right)$. 
2. If $a_{n}$ is a monotonically right $\tau$-convergent sequence to $a$, then $\cap\left(<a_{n}\right)=(\leq a)=\cap\left(\leq a_{n}\right)$.

Proof.

1. Next we prove both equalities:

- $\cup\left(<a_{n}\right)=(<a)$. On the one hand, since $a_{n}<a$, we have that $\left(<a_{n}\right) \subseteq(<a)$. Therefore, $\cup\left(<a_{n}\right) \subseteq(<a)$.

On the other hand, let $x<a$. Since $a_{n} \stackrel{\tau}{\rightarrow} a$ and $a_{n}<a$, there exists $n \in \mathbb{N}$ such that $x<a_{n}<a$ and, hence, $x \in \cup\left(<a_{n}\right)$.

- $\cup\left(<a_{n}\right)=\cup\left(\leq a_{n}\right)$. On the one hand, let $x \in \cup\left(<a_{n}\right)$, then there exists $n \in \mathbb{N}$ such that $x \in\left(<a_{n}\right)$. It is clear that $x \in\left(\leq a_{n}\right)$ and, hence, $x \in \cup\left(\leq a_{n}\right)$.

On the other hand, let $x \in \cup\left(\leq a_{n}\right)$, then there exists $n \in \mathbb{N}$ such that $x \in\left(\leq a_{n}\right)$. Since $a_{n}<a$ and $a_{n} \stackrel{\tau}{\rightarrow} a$, it holds that there exists $m>n$ such that $a_{n}<a_{m}<a$, The fact that $x \in\left(\leq a_{n}\right)$ gives us that $x \in\left(<a_{m}\right)$. We conclude that $x \in \cup\left(<a_{n}\right)$.

2. Next we prove both equalities:

On the one hand, let $x \leq a_{n}$, for each $n \in \mathbb{N}$ and suppose that $x>a$, then there exists $m \in \mathbb{N}$ such that $a<a_{m}<x$, which is a contradiction with the fact that $x \leq a_{n}$, for each $n \in \mathbb{N}$. Hence, $x \leq a$ and $\cap\left(\leq a_{n}\right) \subseteq(\leq a)$.

Moreover, since $a<a_{n}$ for each $n \in \mathbb{N}$, we have that $(\leq a) \subseteq\left(<a_{n}\right)$. Therefore $(\leq a) \subseteq \cap\left(<a_{n}\right)$.

Furthermore, it is clear that $\left(<a_{n}\right) \subseteq\left(\leq a_{n}\right)$, so we conclude that $\cap\left(<a_{n}\right) \subseteq \cap\left(\leq a_{n}\right)$ and we finish the proof.

Proposition 7. Each connected set in $\tau$ is convex.

Proof. Let $A \subseteq X$ be a connected set. Suppose that $A$ is not convex, which means that there exist $a, b \in A$ with $a<b$ such that there exists $x \in X \backslash A$ with $a<x<b$. Please note that $(<x)$ and $(>x)$ are both open sets in $\tau$, which implies that $U=(<x) \cap A$ and $V=(>x) \cap A$ are both open in $A$ with the topology induced by $\tau$ in $A$. Please note that $U, V \neq \varnothing$ since $a \in U, b \in V$ and $U \cup V=A$ which implies that $A$ is not connected. Hence $A$ is convex.

\section{Defining the Distribution Function}

The definition of the cumulative distribution function of a measure defined on the Borel $\sigma$-algebra of $X$ is the next one:

Definition 14. The cumulative distribution function (in short, cdf) of a probability measure $\mu$ is a function $F: X \rightarrow[0,1]$ defined by $F(x)=\mu(\leq x)$.

Lemma 3. Let $\tau^{\prime}$ be a first countable topology on $X$ such that $\tau \subseteq \tau^{\prime}$. Let $f: X \rightarrow[0,1]$ be a monotonically non-decreasing function and $x \in X$ and suppose that $f\left(x_{n}\right) \rightarrow f(x)$ for each monotonically right $\tau^{\prime}$-convergent (respectively monotonically left $\tau^{\prime}$-convergent) sequence to $x$, then $f$ is right $\tau^{\prime}$-continuous (respectively $f$ is left $\tau^{\prime}$-continuous).

Proof. Let $x \in X$ and $x_{n} \stackrel{\tau^{\prime}}{\rightarrow} x$ be a right $\tau^{\prime}$-convergent sequence. If $\left(x_{n}\right)$ is eventually constant (there exists $k \in \mathbb{N}$ such that $x_{n}=x$ for each $n \geq k$ ), the proof is easy. Otherwise, using that $\tau \subseteq \tau^{\prime}$, we can recursively define a decreasing subsequence $x_{\sigma(n)}$ of $x_{n}$, such that $x<x_{\sigma(n+1)}<x_{\sigma(n)}$, for each $n \in \mathbb{N}$.

It follows that $x_{\sigma(n)}$ is monotonically right $\tau^{\prime}$-convergent to $x$ and, hence, by hypothesis, $f\left(x_{\sigma(n)}\right) \rightarrow f(x)$. 
Given $k \in \mathbb{N}$, we have that $x<x_{\sigma(k)}$. Since $\tau \subseteq \tau^{\prime}$ it follows that $x_{n} \stackrel{\tau}{\rightarrow} x$ which gives us that there exists $n_{0} \in \mathbb{N}$, such that $x \leq x_{n}<x_{\sigma(k)}$, for each $n \geq n_{0}$.

Now, the monotonicity of $f$ gives us that $f(x) \leq f\left(x_{n}\right) \leq f\left(x_{\sigma(k)}\right)$, for each $n \geq n_{0}$. We conclude that $f\left(x_{n}\right) \rightarrow f(x)$ and, hence, $f$ is right $\tau^{\prime}$-continuous.

We can proceed analogously to show that $f$ is left $\tau^{\prime}$-continuous when $x_{n}$ is left $\tau^{\prime}$-convergent to $x$.

Corollary 3. Let $\tau^{\prime}$ be a first countable topology on $X$ and $f: X \rightarrow[0,1]$ a function. If $f$ is right and left $\tau^{\prime}$-continuous, then $f$ is $\tau^{\prime}$-continuous.

Proof. Let $x \in X$ and $x_{n} \stackrel{\tau^{\prime}}{\rightarrow} x$. Let $\sigma_{1}, \sigma_{2}: \mathbb{N} \rightarrow \mathbb{N}$ be two increasing functions such that $x_{\sigma_{1}(n)} \geq x$ and $x_{\sigma_{2}(n)} \leq x$ with $\sigma_{1}(\mathbb{N}) \cup \sigma_{2}(\mathbb{N})=\mathbb{N}$. If either $\sigma_{1}(\mathbb{N})$ or $\sigma_{2}(\mathbb{N})$ is finite then the proof is easy. Otherwise, $\left(x_{\sigma_{1}(n)}\right)$ is a right subsequence of $\left(x_{n}\right)$ and $\left(x_{\sigma_{2}(n)}\right)$ is a left subsequence of $\left(x_{n}\right)$. By hypothesis, it holds that $f\left(x_{\sigma_{1}(n)}\right) \rightarrow f(x)$ and $f\left(x_{\sigma_{2}(n)}\right) \rightarrow f(x)$. It easily follows that $f\left(x_{n}\right) \rightarrow f(x)$, which means that $f$ is continuous with respect to the topology $\tau^{\prime}$.

Remark 4. Please note that Lemma 3 and Corollary 3 can be both applied to topology $\tau$.

Corollary 4. Let $\tau^{\prime}$ be a first countable topology on $X$ with $\tau \subseteq \tau^{\prime}$ and let $f: X \rightarrow[0,1]$ be a monotonically non-decreasing function. Suppose that $f\left(x_{n}\right) \rightarrow f(x)$ for each monotonically right $\tau^{\prime}$-convergent sequence to $x$ and each monotonically left $\tau^{\prime}$-convergent sequence, $x_{n}$, to $x$, then $f$ is continuous (with respect to the topology $\left.\tau^{\prime}\right)$.

Proof. It follows from Lemma 3 and Corollary 3.

Proposition 8. Let $F$ be a cdf. Then:

1. $\quad F$ is monotonically non-decreasing.

2. F is right $\tau$-continuous.

3. If there does not exist $\min X$, then $\inf F(X)=0$.

4. $\sup F(X)=1$.

\section{Proof.}

1. This is obvious if we take into account the monotonicity of $\mu$ that follows from the fact that $\mu$ is a measure.

2. For the purpose of proving that $F$ is right $\tau$-continuous, let $x_{n}$ be a monotonically right $\tau$-convergent sequence to $x$. Let us see that $F\left(x_{n}\right) \rightarrow F(x)$.

First, note that the fact that $x_{n}$ is a monotonically right $\tau$-convergent sequence to $x$ implies, by Lemma 2, that $\bigcap_{n}\left(\leq x_{n}\right)=(\leq x)$. Moreover, $\left(\leq x_{n}\right)$ is a monotonically non-increasing sequence so $\left(\leq x_{n}\right) \rightarrow \bigcap_{n}\left(\leq x_{n}\right)=(\leq x)$. Thus, from the continuity of the measure $\mu$, it follows that $\mu\left(\leq x_{n}\right) \rightarrow \mu(\leq x)$, i.e., $F\left(x_{n}\right) \rightarrow F(x)$. Therefore, by Lemma 3 and Remark 4 , we have that $F$ is right $\tau$-continuous.

3. Suppose that there does not exist $\min X$. By Proposition 3, we can consider a sequence $x_{n}$ in $X$ such that $x_{n+1}<x_{n}$, for each $n \in \mathbb{N}$ and $\left\{x_{n}: n \in \mathbb{N}\right\}^{l}=X^{l}=\varnothing$. Then we have $\bigcap\left(\leq x_{n}\right)=\varnothing$. Now, note that $\left(\leq x_{n}\right)$ is a monotonically non-increasing sequence, which implies that $\left(\leq x_{n}\right) \rightarrow$ $\bigcap\left(\leq x_{n}\right)=\varnothing$. By the continuity of the measure $\mu$ it holds that $\mu\left(\leq x_{n}\right)=F\left(x_{n}\right) \rightarrow \mu(\varnothing)=0$. Hence $\inf \left\{F\left(x_{n}\right): n \in \mathbb{N}\right\}=0$. Finally, if we join the previous equality with the fact that $0 \leq \inf F(X) \leq \inf \left\{F\left(x_{n}\right): n \in \mathbb{N}\right\}$, we conclude that $\inf F(X)=0$.

4. We distinguish two cases depending on whether there exists the maximum of $X$ or not:

(a) Suppose that there exists $\max X$. In this case, $\sup F(X)=F(\max X)=\mu(X)=1$. 
(b) Suppose that there does not exist max $X$. By Proposition 3, we can consider a sequence $x_{n}$ in $X$ such that $x_{n+1}>x_{n}$, for each $n \in \mathbb{N}$ and $\left\{x_{n}: n \in \mathbb{N}\right\}^{u}=X^{u}=\varnothing$. Then we have $\mathrm{U}\left(\leq x_{n}\right)=X$. Now, note that $\left(\leq x_{n}\right)$ is a monotonically non-decreasing sequence, which implies that $\left(\leq x_{n}\right) \rightarrow \bigcup\left(\leq x_{n}\right)=X$. By the continuity of the measure $\mu$ it holds that $\mu\left(\leq x_{n}\right)=F\left(x_{n}\right) \rightarrow \mu(X)=1$. Hence $\sup \left\{F\left(x_{n}\right): n \in \mathbb{N}\right\}=1$. Finally, if we join the previous equality with the fact that $\sup \left\{F\left(x_{n}\right): n \in \mathbb{N}\right\} \leq \sup F(X) \leq 1$, we conclude that $\sup F(X)=1$.

The previous proposition makes us wonder the next:

Question 1. Let $F: X \rightarrow[0,1]$ be a function satisfying the properties collected in Proposition 8 , does there exist a probability measure $\mu$ on $X$ such that its $c d f, F_{\mu}$, is $F$ ?

According to the previous results we can conclude that:

Corollary 5. Let $F$ be a cdf and $x \in X$. Then $F$ is $\tau$-continuous at $x$ if, and only if $F$ is left $\tau$-continuous at $x$.

Proposition 9. Let $x \in X$ and $f$ be a monotonically non-decreasing function. If $x$ is left-isolated (respectively right-isolated), then $f$ is left $\tau^{\prime}$-continuous (respectively right $\tau^{\prime}$-continuous) where $\tau^{\prime}$ is a first countable topology such that $\tau \subseteq \tau^{\prime}$.

Proof. Let $x \in X$ and suppose that it is left-isolated. The case in which $x=\min X$ is obvious. Suppose that $x \neq \min X$, then there exists $z \in X$ such that $] z, x[=\varnothing$. Hence $(>z)$ is open in $\tau$ and, consequently, a neighborhood of $x$. Let $x_{n}$ be a left $\tau^{\prime}$-convergent sequence to $x$, then it is also left $\tau$-convergent to $x$. Hence, there exists $n_{0} \in \mathbb{N}$ such that $x_{n} \in(>z)$, for each $n \geq n_{0}$. Since $x_{n} \leq x$, we have that $x_{n}=x$, for each $n \geq n_{0}$. Consequently, $f\left(x_{n}\right) \rightarrow f(x)$ and $f$ is left $\tau^{\prime}$-continuous.

The case in which $x$ is right-isolated can proved analogously.

Corollary 6. Let $\mu$ be a probability measure on $X$ and $F$ its cdf. Let $x \in X$. If $x$ is left-isolated, then $F$ is $\tau$-continuous at $x$.

Proof. It immediately follows from Proposition 9, Corollary 5 and Proposition 8.

Definition 15. Let $\mu$ be a probability measure on $X$ and $F$ its cdf. We define $F_{-}: X \rightarrow[0,1]$, by $F_{-}(x)=$ $\mu(<x)$, for each $x \in X$.

Please note that $F_{-}$is monotonically non-decreasing by the monotonicity of the measure.

Next we introduce two results which relate $F_{-}$with $F$.

Proposition 10. Let $\mu$ be a probability measure on $X$ and $F$ its cdf. Then $\sup F(<x)=F_{-}(x)$, for each $x \in X$ with $x \neq \min X$.

Proof. $\geq$ ) Let $x \in X$ with $x \neq \min X$. We distinguish two cases depending on whether $x$ is left-isolated or not:

1. Suppose that $x$ is not left-isolated, then by Proposition 6, there exists a monotonically left $\tau$-convergent sequence, $a_{n}$, to $x$. This implies that $\left(\leq a_{n}\right) \rightarrow \cup\left(\leq a_{n}\right)$. Moreover, Lemma 2 gives us that $\cup\left(\leq a_{n}\right)=(<x)=\cup\left(<a_{n}\right)$. Hence, $\left(\leq a_{n}\right) \rightarrow(<x)$ and, consequently, $F\left(a_{n}\right) \rightarrow \mu(<x)$. Now, since $a_{n}<x, F\left(a_{n}\right) \leq \sup F(<x)$. If we take limits, we have that $\mu(<x)=F_{-}(x) \leq \sup F(<x)$.

2. Suppose that $x$ is left-isolated, then there exists $z \in X$ such that $z<x$ and $] z, x[=\varnothing$, which implies that $F(z) \leq \sup F(<x)$. Moreover, note that $(<x)=(\leq z)$ which means that $\mu(<x)=F(z)$. We conclude that $\mu(<x)=F_{-}(x) \leq \sup F(<x)$. 
$\leq)$ Let $y \in X$ with $y<x$, then $F(y) \leq \mu(<x)$ and hence $\sup F(<x) \leq \mu(<x)=F_{-}(x)$.

We can recover the cdf $F$ from $F_{-}$.

Proposition 11. Let $F$ be a cdf, then $F(x)=\inf F_{-}(>x)$, for each $x \in X$ with $x \neq \max X$.

Proof. $\leq)$ Let $x \in X$ with $x \neq \max X$ and $y \in X$ be such that $y>x$, then $\mu(<y) \geq \mu(\leq x)$, i.e., $F(x) \leq F_{-}(y)$, which gives us that $F(x) \leq \inf F_{-}(>x)$.

$\geq$ ) Let $x \in X$ with $x \neq \max X$. We distinguish two cases depending on whether $x$ is right-isolated or not.

1. Suppose that $x$ is right-isolated, then there exists $z \in X$ such that $z>x$ and $] x, z[=\varnothing$, which implies that $\inf F_{-}(>x) \leq F_{-}(z)$. Moreover, note that $(>x)=(\geq z)$ which means that $\mu(>x)=\mu(\geq z)$ or, equivalently, $\mu(\leq x)=\mu(<z)$. Hence, $F(x)=F_{-}(z)$. We conclude that $\inf F_{-}(>x) \leq F(x)$.

2. Suppose that $x$ is not right-isolated, then by Proposition 6, there exists a monotonically right $\tau$-convergent sequence, $a_{n}$, to $x$. Since $F$ is right $\tau$-continuous, we have that $F\left(a_{n}\right) \rightarrow F(x)$. Now, the fact that $a_{n}>x$ gives us that inf $F_{-}(>x) \leq F_{-}\left(a_{n}\right) \leq F\left(a_{n}\right)$. Finally, if we take limits, we have that inf $F_{-}(>x) \leq F(x)$.

Lemma 4. Let $\mu$ be a probability measure on $X$ and $F$ its cdf. Given $x \in X$, it holds that $F(x)=F_{-}(x)+$ $\mu(\{x\})$.

Proof. Indeed, given $x \in X$, by the definition of cdf, we have that $F(x)=\mu(\leq x)$. Now, since $\mu$ is $\sigma$-additive, $F(x)=\mu(<x)+\mu(\{x\})$. We conclude that $F(x)=F_{-}(x)+\mu(\{x\})$.

A cdf let us calculate the measure of $] a, b]$ for each $a \leq b$ according to the next proposition and Lemma 4.

Proposition 12. Let $\mu$ be a probability measure on $X$ and $F$ its $c d f$, then $\mu(] a, b])=F(b)-F(a)$ for each $a, b \in X$ with $a<b$.

Proof. Please note that we can write $(\leq b)=(\leq a) \cup] a, b]$. Now, since $\mu$ is a measure (and hence $\sigma$-additive) it holds that $\mu(\leq b)=\mu(\leq a)+\mu(] a, b])$, i.e., $\mu(] a, b])=F(b)-F(a)$.

Corollary 7. Let $\mu$ be a probability measure on $X$ and $F$ its $c d f$, then:

1. $\mu([a, b])=F(b)-F_{-}(a)$.

2. $\mu(] a, b[)=F_{-}(b)-F(a)$.

3. $\mu\left(\left[a, b[)=F_{-}(b)-F_{-}(a)\right.\right.$.

Proof. The proof is immediate if we take into account the previous proposition and Lemma 4.

Proposition 13. Let $\mu$ be a probability measure on $X$ and $F$ its cdf. Let $x \in X$ and $x_{n}$ be a monotonically left $\tau$-convergent sequence to $x$ then $F\left(x_{n}\right) \rightarrow F_{-}(x)$.

Proof. Let $x \in X$ and $x_{n}$ be a monotonically left $\tau$-convergent sequence to $x$. Lemma 2 gives us that $\bigcup_{n}\left(\leq x_{n}\right)=(<x)$. Please note that $\left(\leq x_{n}\right)$ is a monotonically non-decreasing sequence, which means that $\left(\leq x_{n}\right) \rightarrow \bigcup_{n}\left(\leq x_{n}\right)=(<x)$. Finally, by the continuity of $\mu$ it follows that $\mu\left(\leq x_{n}\right) \rightarrow \mu(<x)=F_{-}(x)$, i.e., $F\left(x_{n}\right) \rightarrow F_{-}(x)$.

Next we collect the properties of $F_{-}$: 
Proposition 14. Let $\mu$ be a probability measure on $X$ and $F$ its $c d f$, then:

1. $F_{-}$is monotonically non-decreasing.

2. $F_{-}$is left $\tau$-continuous.

3. $\inf F_{-}(X)=0$.

4. If there does not exist the maximum of $X$, then $\sup F_{-}(X)=1$. Otherwise, $F_{-}(\max X)=1-$ $\mu(\{\max X\})$.

\section{Proof.}

1. This is obvious if we take into account the monotonicity of $\mu$ that follows from the fact that it is a measure.

2. Let $x_{n}$ be a monotonically left $\tau$-convergent sequence to $x$, then by Proposition 13 , it holds that $F\left(x_{n}\right) \rightarrow F_{-}(x)$. Since $x_{n}$ is monotonically left $\tau$-convergent, it holds that $x_{n}<x_{n+1}<x$, so the fact that $F_{-}$is monotonically non-decreasing implies that $F\left(x_{n}\right) \leq F_{-}\left(x_{n+1}\right) \leq F_{-}(x)$. By taking limits, we conclude that $F_{-}\left(x_{n}\right) \rightarrow F_{-}(x)$, and by Lemma 3 and Remark $4, F_{-}$is left $\tau$-continuous.

3. By Proposition 3, we can consider a sequence $x_{n}$ in $X$ such that $x_{n+1}<x_{n}$, for each $n \in \mathbb{N}$ and $\left\{x_{n}: n \in \mathbb{N}\right\}^{l}=X^{l}=\varnothing$. Then we have $\bigcap\left(<x_{n}\right)=\varnothing$. Now, note that $\left(<x_{n}\right)$ is a monotonically non-increasing sequence, which implies that $\left(<x_{n}\right) \rightarrow \bigcap\left(<x_{n}\right)=\varnothing$. By the continuity of the measure $\mu$ it holds that $\mu\left(<x_{n}\right)=F_{-}\left(x_{n}\right) \rightarrow \mu(\varnothing)=0$. Hence $\inf \left\{F_{-}\left(x_{n}\right): n \in \mathbb{N}\right\}=0$. Finally, if we join the previous equality with the fact that $0 \leq \inf F_{-}(X) \leq \inf \left\{F_{-}\left(x_{n}\right): n \in \mathbb{N}\right\}$, we conclude that $\inf F_{-}(X)=0$.

4. We distinguish two cases depending on whether there exists the maximum of $X$ or not:

(a) Suppose that there does not exist $\max X$. By Proposition 3, there exists a sequence $x_{n}$ in $X$ such that $x_{n+1}>x_{n}$, for each $n \in \mathbb{N}$ and $\left\{x_{n}: n \in \mathbb{N}\right\}^{u}=X^{u}=\varnothing$. Then we have $\mathrm{U}\left(<x_{n}\right)=X$. Now, note that $\left(<x_{n}\right)$ is a monotonically non-decreasing sequence, which implies that $\left(<x_{n}\right) \rightarrow \bigcup\left(<x_{n}\right)=X$. By the continuity of the measure $\mu$ it holds that $\mu\left(<x_{n}\right)=F_{-}\left(x_{n}\right) \rightarrow \mu(X)=1$. Hence $\sup \left\{F_{-}\left(x_{n}\right): n \in \mathbb{N}\right\}=1$. Finally, if we join the previous equality with the fact that $\sup \left\{F_{-}\left(x_{n}\right): n \in \mathbb{N}\right\} \leq \sup F_{-}(X) \leq 1$, we conclude that $\sup F_{-}(X)=1$.

(b) Now suppose that there exists $\max X$, then Lemma 4 let us claim that $F_{-}(\max X)=$ $F(\max X)-\mu(\{\max X\})=1-\mu(\{\max X\})$.

Thus, the item is proved.

\section{Discontinuities of a cdf}

In this section, we prove some results which are analogous to those proven in Chapter 1 of [18] and which are related to the discontinuities of a cdf.

First, we give a sufficient condition to ensure that a cdf is continuous at a point.

Proposition 15. Let $x \in X, \mu$ be a probability measure on $X$ and $F$ its cdf. If $\mu(\{x\})=0$ then $F$ is $\tau$-continuous at $x$.

Proof. Let $x_{n}$ be a monotonically left $\tau$-convergent sequence to $x$, then by Proposition 13, it holds that $F\left(x_{n}\right) \rightarrow F_{-}(x)$. By Lemma 4, it holds that $F(x)=F_{-}(x)$, so $F\left(x_{n}\right) \rightarrow F(x)$, and by Lemma 3 and Remark $4, F$ is left $\tau$-continuous. Finally, by Corollary $5, F$ is $\tau$-continuous.

Next we introduce a lemma that will be crucial to show that the set of discontinuity points of a cdf is at most countable. 
Lemma 5. Let $\mu$ be a probability measure on $X$ and $F$ its cdf. Then $\{x \in X: \mu(\{x\})>0\}$ is countable.

Proof. For every integer $N$, the number of points satisfying $\mu(\{x\})>\frac{1}{N}$ is, at most, $N$. Hence, there are no more than a countable number of points with positive measure.

Next we collect two properties of a cdf $F_{\mu}$.

Proposition 16. Let $\mu$ be a probability measure on $X$, then

1. $F_{\mu}$ is determined by a dense set, $D$, in $X$ (with respect to the topology $\tau$ ) in its points with null measure, that is, if for each $x \in D$ it holds that $F_{\mu}(x)=F_{\delta}(x)$, then $F_{\mu}(x)=F_{\delta}(x)$, for each $x \in X$ with $\mu(\{x\})=0$ and $\delta(\{x\})=0$, where $F_{\delta}$ is the cdf of a probability measure, $\delta$, on $X$.

2. The set of discontinuity points of $F_{\mu}$ with respect to the topology $\tau$ is countable.

\section{Proof.}

1. Let $x \in X$ with $\mu(\{x\})=0$ and $\delta(\{x\})=0$. We distinguish two cases:

- $\quad$ Suppose that $x$ is left-isolated and right-isolated, then there exist $y, z \in X$ such that $] y, z[=$ $\{x\}$, which implies that $x \in D$ due to the fact that $D$ is dense. Consequently, $F_{\mu}(x)=F_{\delta}(x)$.

- $\quad x$ is not left-isolated or it is not right-isolated. If $x$ is not left-isolated, by Proposition 6, there exists a sequence $x_{n} \stackrel{\tau}{\rightarrow} x$ such that $x_{n}<x_{n+1}<x$. Now, since $D$ is dense, it follows that there exists $d_{n} \in D$ such that $x_{n}<d_{n}<x_{n+1}$ and hence $d_{n}<d_{n+1}$, for each $n \in \mathbb{N}$. Hence, $d_{n} \rightarrow x$ in $\tau$. By hypothesis, we have that $F_{\mu}\left(d_{n}\right)=F_{\delta}\left(d_{n}\right)$. By Proposition 13, $F_{\mu}\left(d_{n}\right) \rightarrow F_{\mu-}(x)$. However, $F_{\mu-}(x)=F_{\mu}(x)$ since $\mu(\{x\})=0$ by Lemma 4. Analogously, $F_{\delta}\left(d_{n}\right) \rightarrow F_{\delta}(x)$. Consequently, $F_{\mu}(x)=F_{\delta}(x)$.

The case in which $x$ is not right-isolated can be proved analogously.

2. Let $x \in X$. By Proposition 15, we know that the fact that $F_{\mu}$ is not continuous at $x$ means that $\mu(\{x\})>0$. Since, by previous lemma, we have that $\{x \in X: \mu(\{x\})>0\}$ is countable, we conclude that the set of discontinuity points is at most countable too.

\section{The Inverse of a cdf}

In this section, we see how to define the pseudo-inverse of a cdf $F$ defined on $X$ and we gather some properties which relate this function to both $F$ and $F_{-}$. Its properties are similar to those which characterizes the pseudo-inverse in the classical case (see, for example, [1], Th. 1.2.5). Moreover, we see that it is measurable.

Now, we recall the definition of this function in the classical case (see Section 1) to give a similar one in the context of a linearly ordered topological space. However, there exists a problem when we mention the infimum of a set, since there is no guarantee that every set has infimum. Indeed, it is possible to extend the cdf to the Dedekind-MacNeille completion so that the pseudo-inverse is naturally defined from $[0,1]$ to the Dedekind-MacNeille completion as it can be seen in [19]. Hence, in this work, we restrict that definition to those points which let us talk about the infimum of a set as next definition shows.

Definition 16. Let $F$ be a cdf. We define the pseudo-inverse of $F$ as $G:[0,1] \rightarrow X$ given by $G(x)=\inf \{y \in$ $X: F(y) \geq x\}$ for each $x \in[0,1]$ such that there exists the infimum of $\{y \in X: F(y) \geq x\}$.

According to the previous definition, it is clear that

Proposition 17. G is monotonically non-decreasing. 
Proof. Let $x, y \in[0,1]$ with $x<y$. Please note that $\{z \in X: F(z) \geq y\} \subseteq\{z \in X: F(z) \geq x\}$ and it follows that $\inf \{z \in X: F(z) \geq x\} \leq \inf \{z \in X: F(z) \geq y\}$, i.e., $G(x) \leq G(y)$, which means that $G$ is monotonically non-decreasing.

Lemma 6. Let $a=\inf \left\{a_{n}: n \in \mathbb{N}\right\}$ (respectively $a=\sup \left\{a_{n}: n \in \mathbb{N}\right\}$ ) where $a_{n}$ is a sequence such that $a_{n+1}<a_{n}$ (respectively $\left.a_{n+1}>a_{n}\right)$, for each $n \in \mathbb{N}$. Then $a_{n} \stackrel{\tau}{\rightarrow} a$.

Proof. Let $a_{n}$ be a sequence in $X$ such that $a_{n+1}<a_{n}$, for each $n \in \mathbb{N}$ and suppose that there exists $a=\inf \left\{a_{n}: n \in \mathbb{N}\right\}$. Let $b, c \in X \cup\{-\infty, \infty\}$ be such that $b<a<c$. Suppose that $a_{n} \geq c$, for each $n \in \mathbb{N}$, then $\inf \left\{a_{n}: n \in \mathbb{N}\right\} \geq c>a$, a contradiction with the fact that $a=\inf \left\{a_{n}: n \in \mathbb{N}\right\}$. Hence, there exists $n_{0} \in \mathbb{N}$ such that $a_{n_{0}}<c$. Furthermore, $a_{n}<c$, for each $n \geq n_{0}$ since $a_{n+1}<a_{n}$, for each $n \in \mathbb{N}$. Consequently, $a_{n} \stackrel{\tau}{\rightarrow} a$.

The case in which $a=\sup \left\{a_{n}: n \in \mathbb{N}\right\}$ and $a_{n+1}>a_{n}$ can be proven analogously.

Hereinafter, when we apply $G$ to a point, we assume that $G$ is defined in that point.

Proposition 18. Let $F$ be a cdf. Then:

1. $G(F(x)) \leq x$, for each $x \in X$.

2. $F(G(r)) \geq r$, for each $r \in[0,1]$.

\section{Proof.}

1. Indeed, $x \in\{z \in X: F(z) \geq F(x)\}$, and hence $\inf \{z \in X: F(z) \geq F(x)\} \leq x$, which is equivalent to $G(F(x)) \leq x$. This proves the first item.

2. Now let $y=G(r)=\inf \{z \in X: F(z) \geq r\}$. If $y=\min \{z \in X: F(z) \geq r\}$, it is clear that $F(y) \geq r$. Suppose that $y \neq \min \{z \in X: F(z) \geq r\}$, then by Proposition 3 there exists a sequence $y_{n} \in\{z \in X: F(z) \geq r\}$ such that $y_{n+1}<y_{n}$ and $\left\{y_{n}: n \in \mathbb{N}\right\}^{l}=\{z \in X: F(z) \geq r\}^{l}$. Furthermore, by Lemma 1 , it holds that $y=\inf \left\{y_{n}: n \in \mathbb{N}\right\}$. Hence, Lemma 6 let us claim that $y_{n} \stackrel{\tau}{\rightarrow} y$. Consequently, the right $\tau$-continuity of $F$ gives us that $F\left(y_{n}\right) \rightarrow F(y)$. Moreover, $F\left(y_{n}\right) \geq r$ since $y_{n} \in\{z \in X: F(z) \geq r\}$. If we join this fact with the fact that $F\left(y_{n}\right) \rightarrow F(y)$, we conclude that $F(y) \geq r$. This proves the second item.

We get, as an immediate corollary, that

Corollary 8. $G(r) \leq x$ if, and only if $r \leq F(x)$, for each $x \in X$ and each $r \in[0,1]$.

Next result collects some properties of $G$ which arise from some relationships between $F$ and $F_{-}$ and some conditions on them.

Proposition 19. Let $F$ be a cdf and let $x \in X$ and $r \in[0,1]$. Then:

1. $F(x)<r$ if, and only if $G(r)>x$.

2. If $F_{-}(x)<r$, then $x \leq G(r)$.

3. If $F_{-}(x)<r \leq F(x)$, then $G$ is defined in $r$ and $G(r)=x$.

4. If $r<F_{-}(x)$, then $G(r)<x$.

5. If $r=F_{-}(x)$, then $G(r) \leq x$.

Proof.

1. Please note that it is an immediate consequence of Corollary 8 .

2. Suppose that $G(r)<x$, then $\mu(<x) \geq \mu(\leq G(r))$ or, equivalently, $F_{-}(x) \geq F(G(r)) \geq r$, i.e., $F_{-}(x) \geq r$. 
3. Let $x \in X$ and $r \in[0,1]$ be such that $F_{-}(x)<r \leq F(x)$. First, note that if $y<x$, then $F(y) \leq \sup F(<x)=F_{-}(x)<r$ and hence $x=\inf \{y \in X: F(y) \geq r\}$. It follows that $G$ is defined in $r$ and $x=G(r)$.

4. Let $x \in X$ and $r \in[0,1]$. Suppose that $r<F_{-}(x)$. Since $F_{-}(x)=\sup F(<x)$, there exists $y<x$ such that $r<F(y) \leq F_{-}(x)$. Since $F(y)>r$, then $y \geq \inf \{z \in X: F(z) \geq r\}=G(r)$. We conclude that $G(r)<x$.

5. Suppose that $r=F_{-}(x)$. The fact that $F_{-}(x) \leq F(x)$, for each $x \in X$ gives us that $F(x) \geq r$, which is equivalent, by Corollary 8 , to $G(r) \leq x$.

We prove another property of $G$.

Proposition 20. $G$ is left $\tau$-continuous.

Proof. Let $\left(r_{n}\right)$ be a sequence in $\left[0,1\right.$ [ which is left convergent to $r \in\left[0,1\left[\right.\right.$ with $r_{n} \neq r$. Since $r_{n} \leq r$, by the monotonicity of $G$ (see Proposition 17) we have that $G\left(r_{n}\right) \leq G(r)$. Now we prove that $G(r)=\sup \left\{G\left(r_{n}\right): n \in \mathbb{N}\right\}$. For this purpose, let $x \in\left\{G\left(r_{n}\right): n \in \mathbb{N}\right\}^{u}$ and suppose that $x<G(r)$. By Proposition 19, it holds that $F(x)<r$, so there exists $n \in \mathbb{N}$ such that $F(x)<r_{n}$. On the other hand, since $x \in\left\{G\left(r_{n}\right): n \in \mathbb{N}\right\}^{u}$ then $G\left(r_{n}\right) \leq x$, for each $n \in \mathbb{N}$. By the monotonicity of $F$ we have that $F\left(G\left(r_{n}\right)\right) \leq F(x)$ and, hence, by Proposition 18, $r_{n} \leq F(x)$ since $F\left(G\left(r_{n}\right)\right) \geq r_{n}$. If we join this fact with the fact that $F(x)<r_{n}$, for some $n \in \mathbb{N}$, we conclude that $r_{n}<r_{n}$, a contradiction.

It follows, by Lemma 6 , that $\left(G\left(r_{n}\right)\right) \tau$-converges to $G(r)$.

Next proposition collects some properties of $F$ and $F_{-}$which arise from considering some conditions on $G$.

Proposition 21. Let $F$ be a cdf and let $x \in X$ and $r \in[0,1]$. Then:

1. $G(r)>x$ if, and only if $F(x)<r$.

2. If $G(r)=x$, then $F_{-}(x) \leq r \leq F(x)$.

3. If $G(r)<x$, then $r \leq F_{-}(x)$.

\section{Proof.}

1. Please note that this item is the same as the first item of Proposition 19.

2. Suppose that $G(r)=x$ and that $r>F(x)$, by item 1 it follows that $G(r)>x$, which is a contradiction with the fact that $G(r)=x$.

Now suppose that $r<F_{-}(x)$, then item 4 of Proposition 19 gives us that $G(r)<x$, which is a contradiction with the fact that $G(r)=x$.

We conclude that $F_{-}(x) \leq r \leq F(x)$.

3. It is equivalent to Proposition 19.

Some consequences that arise from the previous propositions are collected next.

Corollary 9. Let $F$ be $a$ cdf and $r \in[0,1]$. Then:

1. $F_{-}(G(r)) \leq r \leq F(G(r))$.

2. If $F(G(r))>r$, then $\mu(\{G(r)\})>0$. 


\section{Proof.}

1. Let $r \in[0,1]$. On the one hand, suppose that $F_{-}(G(r))>r$, then, by item 4 of Proposition 19, it holds that $G(r)<G(r)$, which is a contradiction. Hence, $F_{-}(G(r)) \leq r$.

On the other hand, the inequality $r \leq F(G(r))$ is clear if we take into account Proposition 18.

2. By Lemma $4 F(x)=F_{-}(x)+\mu(\{x\})$, for each $x \in X$, so we have that $F(G(r))=F_{-}(G(r))+$ $\mu(\{G(r)\})$. If $F(G(r))>r$, it holds that $F_{-}(G(r))+\mu(\{G(r)\})>r$. Moreover, if we join this fact with the previous item, we conclude that $\mu(\{G(r)\})>0$.

Corollary 10. Let $r \in[0,1]$. If $\mu(\{G(r)\})=0$, then $F(G(r))=r$.

Now, we introduce some results to characterize the injectivity of $G$ and $F$.

Proposition 22. $\mu(\{x\})=0$, for each $x \in X$ if, and only if $G$ is injective.

Proof. $\Rightarrow$ ) It immediately follows from the second item of Proposition 21. Indeed, this proposition gives us that if $G(r)=x$, then $F_{-}(x) \leq r \leq F(x)$. Suppose that there exists $r, s \in X$ such that $r \neq s$ with $G(r)=G(s)=x$, then $F_{-}(G(r)) \leq r \leq F(G(r))$ and $F_{-}(G(r)) \leq s \leq F(G(r))$. Since $\mu(\{G(r)\})=0$, it holds that $F_{-}(G(r))=F(G(r))=r=s$, and hence $G$ is injective.

$\Leftarrow)$ Suppose that there exists $x \in X$ such that $\mu(\{x\})>0$, then $F_{-}(x)<F(x)$. Now let $r \in[0,1]$ be such that $F_{-}(x)<r<F(x)$. By Proposition 19 we have that $G$ is defined in $r$ and $G(r)=G(F(x))=x$, for each $r \in] F_{-}(x), F(x)$ [, which is a contradiction with the fact that $G$ is injective.

Proposition 23. Let $F$ be $a$ cdf, then $F$ is injective if, and only if $\mu(] a, b])>0$, for each $a<b$.

Proof. Let $a, b \in X$ be such that $a<b$. Please note that by Proposition $12, \mu(] a, b])=0$ is equivalent to $F(b)-F(a)=0$, i.e., $F(b)=F(a)$ if, and only if $F$ is not injective.

And we get, as immediate corollary, the next one:

Corollary 11. Let $F$ be a cdf of a probability measure $\mu$, and let $A \subseteq[0,1]$ be the subset of points where $G$ is defined. The following statements are equivalent:

1. $F \circ G(r)=r$ for each $r \in A, F(X) \subseteq A$ and $G \circ F(x)=x$ for each $x \in X$.

2. $F$ is injective and $F(X)=A$.

3. $G: A \rightarrow X$ is bijective.

4. $\mu(] a, b])>0$, for each $a<b$ and $\mu(\{a\})=0$, for each $a \in X$.

Proof. First, we prove the following

Claim. If $F$ is injective, then $F(X) \subseteq A$ and $G(F(x))=x$ for each $x \in X$.

Suppose that there exists $x \in X$ such that $G$ is not defined in $F(x)$, i.e., there does not exists the infimum of $\{y \in X: F(y) \geq F(x)\}$. It follows that $x$ is not the infimum of the latter set, so there exists $y<x$ with $F(y) \geq F(x)$. By monotonicity of $F$ it follows that $F(y)=F(x)$, and since $F$ is injective $y=x$, a contradiction. We conclude that $F(X) \subseteq A$.

Finally, let $x \in X$, then $F(x) \in A$ and $G(F(x))=\inf \{y \in X: F(y) \geq F(x)\}$. On the other hand, if $y<x$ then $F(y) \leq F(x)$, and since $F$ is injective $F(y)<F(x)$. Therefore $G(F(x))=x$.

$(1) \Longrightarrow(2)$. Since $F(X) \subseteq A$ and $G(F(x))=x$ for each $x \in X$, it follows that $F$ is injective. Now, we prove that $A \subseteq F(X)$. Indeed, let $r \in A$, then $F(G(r))=r$, so $r \in F(X)$.

$(1) \Longrightarrow(3)$. Since $G(F(x))=x$ for each $x \in X$, it follows that $G$ is surjective. Since $F(G(r))=r$ for each $r \in A$, it follows that $G$ is injective. 
$(1) \Longrightarrow(4)$. Since (1) implies (2) and (3), we have that $F$ and $G$ are both injective, so (4) follows from Propositions 22 and 23.

(2) $\Longrightarrow(1)$. Let $r \in A$. Since $F(X)=A$ and $F$ is injective, there is only one $x \in X$ such that $F(x)=r$. It follows by definition of $G$ that $G(r)=x$ and hence $F(G(r))=F(x)=r$. By the claim we have the rest of item (1).

(3) $\Longrightarrow(1)$. Let $r \in A$, then $F(G(r)) \geq r$ by Proposition 18 . Suppose that $F(G(r))>r$. It easily follows that $] r, F(G(r))[\subseteq A$ and $G(] r, F(G(r))[)=G(r)$, but this is a contradiction, since $G$ is injective. We conclude that $F(G(r))=r$.

Now, let $x \in X$. Since $G$ is bijective, there exists $r \in A$ such that $x=G(r)$. It follows that $F(x)=F(G(r))=r$ and hence $F(x) \in A$. Therefore $F(X) \subseteq A$.

Finally, let $x \in X$, then $F(x) \in A$ and $G(F(x))=\inf \{y \in X: F(y) \geq F(x)\}$. Suppose that there exists $y<x$ such that $F(y) \geq F(x)$. By monotonicity of $F$ it follows that $F(y)=F(x)$. Since $G$ is bijective, there exists $r, s \in[0,1]$ such that $G(r)=y$ and $G(s)=x$. Please note that $r<s$ by monotonicity of $G$. It follows that $r=F(G(r))=F(y)=F(x)=F(G(s))=s$, a contradiction. We conclude that $x=\inf \{y \in X: F(y) \geq F(x)\}=G(F(x))$.

$(4) \Longrightarrow(1)$. By Corollary 10 it follows that $F(G(r))=r$ for each $r \in A$. By Proposition 23, $F$ is injective and by the claim it follows that $F(X) \subseteq A$ and $G(F(x))=x$ for each $x \in X$.

Proposition 24. Let $a, b \in X$ be such that $a<b$, then $\left.G^{-1}(] a, b[)=\right] F(a), F_{-}(b) \mid \cap A$, where $\mid$ means ] or [ and $A$ is the subset of $[0,1]$ where $G$ is defined.

Proof. First, we show that $\left.\left.G^{-1}(] a, b[) \subseteq\right] F(a), F_{-}(b)\right] \cap A$. For that purpose, let $r \in G^{-1}(] a, b[)$ and suppose that $\left.r \notin] F(a), F_{-}(b)\right]$, then it can happen:

- $\quad r \leq F(a)$ which implies, by Corollary 8 , that $G(r) \leq a$ which is a contradiction with the fact that $r \in G^{-1}(] a, b[)$.

- $\quad r>F_{-}(b)$ which gives us, by Proposition 19, that $b \leq G(r)$ which implies that $r \notin G^{-1}(] a, b[)$ since $b \notin] a, b[$, a contradiction.

Now we prove that $] F(a), F_{-}(b)\left[\cap A \subseteq G^{-1}(] a, b[)\right.$. For that purpose, let $\left.r \in\right] F(a), F_{-}(b)[$ where $G$ is defined, and suppose that $r \notin G^{-1}(] a, b[)$, then it can happen:

- $G(r) \leq a$ which implies, by Corollary 8, that $r \leq F(a)$, a contradiction with the fact that $r>F(a)$.

- $G(r) \geq b$ which gives us, by Proposition 19, that $r \geq F_{-}(b)$, a contradiction with the fact that $r<F_{-}(b)$.

We conclude that $r \in G^{-1}(] a, b[)$.

According to Proposition 24, it is clear the next

Corollary 12. Suppose that $G$ is defined on $[0,1]$. Let $a, b \in X$ be such that $a<b$. Then $G^{-1}(] a, b[) \in$ $\sigma([0,1])$, where $\sigma([0,1])$ denotes de Borel $\sigma$-algebra with respect to the Euclidean topology.

Proof. Since $\left.G^{-1}(] a, b[)=\right] F(a), F_{-}(b) \mid$, it is an open set or the intersection of an open and a closed set. Consequently, $G^{-1}(] a, b[) \in \sigma([0,1])$.

Proposition 25. Each open set in $\tau$ is the countable union of open intervals.

Proof. Let $G \subseteq X$ be an open set in $\tau$. If $G=\varnothing$, the result is clear since it can be written as $G=] a, a[$. Now suppose that $G$ is non-empty, then $G=\bigcup_{i \in I} G_{i}$, where $G_{i}$ is a convex component of $G$ for each $i \in I$ (see Proposition 1). Now we prove that $G_{i}$ is open for each $i \in I$. Let $i \in I$ and $x \in G_{i}$. Since $G$ is an open set and \{]$a, b[: a, b \in X, a<b\}$ is an open basis of $X$ with respect to $\tau$ there exist $a, b \in X$ such that $x \in] a, b\left[\subseteq G\right.$. Please note that $\left.G_{i} \cup\right] a, b[$ is a convex set contained in $G$, which implies that $\left.G_{i} \cup\right] a, b\left[=G_{i}\right.$ since $G_{i}$ is a convex component of $G$. Consequently, $] a, b\left[\subseteq G_{i}\right.$, which means that $G_{i}$ is 
an open set. Now, let $D$ be a countable dense subset of $X$, then we can choose $d_{i} \in D \cap G_{i}$ for each $i \in I$ which gives us the countability of $I$, since the family $\left\{G_{i}: i \in I\right\}$ is pairwise disjoint.

Since $G_{i}$ is convex and open, by Corollary $2, G_{i}$ can be expressed as a countable union of open intervals. Thus, $G$ is the countable union of open intervals.

Next result will be essential to show that $G$ is measurable with respect to the Borel $\sigma$-algebra.

Theorem 1 ([20], Th. 1.7.2). Let $(\Omega, \mathfrak{A})$ and $\left(\Omega^{\prime}, \mathfrak{A}^{\prime}\right)$ be measurable spaces; further let $\mathfrak{B}^{\prime}$ be a generator of $\mathfrak{A}^{\prime}$. A mapping $T: \Omega \rightarrow \Omega^{\prime}$ is measurable if, and only if $T^{-1}\left(A^{\prime}\right) \in \mathfrak{A}$, for each $A^{\prime} \in \mathfrak{B}^{\prime}$.

Since $G^{-1}(] a, b[) \in \sigma([0,1])$, for each $a, b \in X$ with $a<b$ and by taking into account Proposition 25, we conclude that

Corollary 13. Suppose that $G$ is defined on $[0,1]$. Then $G$ is measurable with respect to the Borel $\sigma$-algebras.

Proof. To show that $G$ is measurable we just have to use Corollary 12, Theorem 1 and the fact that each open set in $\tau$ can be written as countable union of open intervals (see Proposition 25).

\section{Generating Samples}

Lemma 7. The family $\mathfrak{A}=\left\{\bigcup_{i=1}^{n}\left|a_{i}, b_{i}\right|: a_{1} \leq b_{1}<a_{2} \leq b_{2}<\ldots<a_{n} \leq b_{n}, a_{1} \in X \cup\{-\infty\}, b_{n} \in\right.$ $X \cup\{\infty\}\}$ is an algebra and the $\sigma$-algebra generated by it is the Borel $\sigma$-algebra.

Proof. Now we prove that $\mathfrak{A}$ is an algebra.

1. $A \cup B \in \mathfrak{A}$, for each $A, B \in \mathfrak{A}$. Indeed, this is true because the union of two intervals consists of two disjoint intervals in case $A \cap B=\varnothing$ or it is a new interval otherwise.

2. $A \cap B \in \mathfrak{A}$, for each $A, B \in \mathfrak{A}$. Indeed, this is true because the intersection of two intervals is $\varnothing$ or a new interval. Hence, $A \cap B$ is finite union of disjoint intervals, which means that $A \cap B \in \mathfrak{A}$.

3. $X \backslash A \in \mathfrak{A}$, for each $A \in \mathfrak{A}$. Indeed, this is true due to the fact that $X \backslash A=]-\infty, a_{1}|\cup| b_{1}, a_{2} \mid \cup$ $\ldots \cup\left|b_{n-1}, a_{n}\right| \cup \mid b_{n}, \infty[\in \mathfrak{A}$.

Please note that each element in $\mathfrak{A}$ belongs to $(X, \tau)$. Indeed, this is true due to the fact that given $A \in \mathfrak{A}$, it consists of the finite union of open intervals, semi-open intervals (which are the intersection of an open and a closed set) or closed intervals (which are closed). Hence, $\mathfrak{S}$ is contained in the Borel $\sigma$-algebra of $(X, \tau)$, where $\mathfrak{S}=\sigma(\mathfrak{A})$. Finally, if $G$ is an open set in $(X, \tau)$, by Proposition 25 , it can be written as the countable union of open intervals. Thus, $G$ can be written as the countable union of elements in $\mathfrak{A}$, which means that $G \in \mathfrak{S}$. In conclusion, $\mathfrak{S}$ is the Borel $\sigma$-algebra of $(X, \tau)$.

Now, we want to prove the uniqueness of the measure with respect to its cdf.

First, we recall from [12] a theorem about the uniqueness of a measure. As a consequence of the next theorem we have that two measures that coincide in an algebra also coincide in its generated $\sigma$-algebra.

Theorem 2 ([12], Chapter III, Th. A). If $\mu$ is a $\sigma$-finite measure on a ring $R$, then there is a unique measure $\bar{\mu}$ on the $\sigma$-ring $S(R)$ such that for $E$ in $R, \bar{\mu}(E)=\mu(E)$; the measure $\bar{\mu}$ is $\sigma$-finite.

Proposition 26. Let $F_{\mu}$ and $F_{\delta}$ be the CDFs of the measures $\mu$ and $\delta$ satisfying $F_{\mu}=F_{\delta}$, then $\mu=\delta$ on the Borel $\sigma$-algebra of $(X, \tau)$.

Proof. Let $a, b \in X$ be such that $a \leq b$, then a cdf let us determine the measure of the set $|a, b|$. Indeed, we distinguish four cases depending on whether $a$ and $b$ belongs to $|a, b|$ or not.

1. $\left.\left.\mu(] a, b])=F_{\mu}(b)-F_{\mu}(a)=F_{\delta}(b)-F_{\delta}(a)=\delta(] a, b\right]\right)$. 
2. $\quad \mu([a, b])=F_{\mu}(b)-F_{\mu-}(a)=F_{\mu}(b)-\sup F_{\mu}(<a)=F_{\delta}(b)-\sup F_{\delta}(<a)=F_{\delta}(b)-F_{\delta-}(a)=$ $\delta([a, b])$, where we have taken into account that $F_{-}(x)=\sup F(<x)$, for each $x \in X$ (see Proposition 10).

3. $\mu(] a, b[)=F_{\mu-}(b)-F_{\mu}(a)=\sup F_{\mu}(<b)-F_{\mu}(a)=\sup F_{\delta}(<b)-F_{\delta}(a)=F_{\delta-}(b)-F_{\delta}(a)=$ $\delta(] a, b[)$.

4. $\quad \mu\left(\left[a, b[)=F_{\mu-}(b)-F_{\mu-}(a)=\sup F_{\mu}(<b)-\sup F_{\mu}(<a)=\sup F_{\delta}(<b)-\sup F_{\delta}(<a)=\right.\right.$ $F_{\delta_{-}}(b)-F_{\delta_{-}}(a)=\delta([a, b[)$.

Since $\mu(|a, b|)=\delta(|a, b|)$, for each $a, b \in X$ with $a \leq b$, it follows that $\mu(A)=\delta(A)$, for each $A \in \mathfrak{A}$, due to the $\sigma$-additivity of $\mu$ and $\delta$ as measures. Since $\mu=\delta$ on $\mathfrak{A}$, we conclude that $\mu=\delta$ on $\sigma(\mathfrak{A})$, i.e., they coincide on the Borel $\sigma$-algebra of $(X, \tau)$ by the previous results.

Theorem 3 ([21], Th A. 81). A measurable function $f$ from one measure space $\left(S_{1}, \mathcal{A}_{1}, \mu_{1}\right)$ to a measurable space $\left(S_{2}, \mathcal{A}_{2}\right), f: S_{1} \rightarrow S_{2}$, induces a measure on the range $S_{2}$. For each, $A \in \mathcal{A}_{2}$, define $\mu_{2}(A)=$ $\mu_{1}\left(f^{-1}(A)\right)$. Integrals with respect to $\mu_{2}$ can be written as integrals with respect to $\mu_{1}$ in the following way if $g: S_{2} \rightarrow \mathbb{R}$ is integrable, then,

$$
\int g(y) d \mu_{2}(y)=\int g(f(x)) d \mu_{1}(x)
$$

Proposition 27. Let $\mu$ be a probability measure and suppose that $G$ is defined on $[0,1]$. Then $\mu(A)=$ $l\left(G^{-1}(A)\right)$ for each $A \in \sigma([0,1])$, where $l$ is the Lebesgue measure and $\sigma([0,1])$ is the Borel $\sigma$-algebra of $[0,1]$.

Proof. By Proposition 24, we have that $\left.G^{-1}(] a, b[)=\right] F(a), F_{-}(b) \mid$, for each $a, b \in X$ with $a<b$.

Moreover, by Corollary 7 , it holds that $\mu(] a, b[)=F_{-}(b)-F(a)$. It follows that $l\left(G^{-1}(] a, b[)\right)=$ $\mu(] a, b[)$ for each $a, b \in X$ with $a<b$.

Now let $\mu_{2}$ be the measure defined by $\mu_{2}(A)=l\left(G^{-1}(A)\right)$, for each $A \in \sigma([0,1])$. Indeed, $\mu_{2}$ is a measure by Theorem 3 and Corollary 13. Please note that the fact that $\mu(] a, b[)=\mu_{2}(] a, b[)$, for each $a, b \in X$ with $a<b$, implies that $\mu=\mu_{2}$ on the algebra $\mathfrak{A}$. Therefore $\mu$ and $\mu_{2}$ coincides in an algebra which generates $\sigma([0,1])$, so they are equal in $\sigma([0,1])$ (see for example Theorem 2 ).

Consequently, we can write $\mu(A)=l\left(G^{-1}(A)\right)$ for each $A \in \sigma([0,1])$.

Finally, by taking into account the previous results, we can generate samples with respect to the probability measure $\mu$ by following the classical procedure (see Section 1 ). In our case we will have to use $G$ to do it.

Remark 5. Suppose that $G$ is defined on $[0,1]$. We can also calculate integrals with respect to $\mu$ by using Theorem 3, so for $g: X \rightarrow \mathbb{R}$,

$$
\int g(x) d \mu(x)=\int g(G(t)) d t
$$

Remark 6. Suppose that $X$ is compact, then every subset of $X$ has both infimum and supremum (see Proposition 2) and hence $G$ is defined in each point of $[0,1]$. Therefore, in this case, we can generate samples with respect to a distribution based on a measure $\mu$.

Remark 7. Please note that the classical theory for the distribution function is a particular case of the one we have developed for a separable LOTS.

\section{Conclusions}

This paper proposes a theory of distribution functions on linearly ordered topological spaces. Indeed, the classical theory, which is well-known in case we define a probability measure on $\mathbb{R}$, is a particular case of the one described in this work. The developed theory is similar to the one known 
in several aspects. For example, a cumulative distribution function on a LOTS, $X$, is non-decreasing, right-continuous (with respect to the order topology) and $\sup F(X)=1$ (see Proposition 8). However, the fact that $\inf F(X)=0$ can only be ensured in case there does not exist the minimum of $X$.

It is also worthy to be highlighted the fact that the measure of each point being 0 is not equivalent to the continuity of the cdf, as it happens in the classical case (see Proposition 15). Furthermore, a step cdf can be continuous, while it cannot happen in the classical case.

From $F$, it is possible to define a function, that we call $F_{-}$(see Definition 15), that plays a similar role to that played by the $\operatorname{limit}_{\lim _{x \rightarrow a^{-}}} F(x)$ in the classical case (see Proposition 4). Indeed, both $F$ and $F_{-}$let us calculate the measure of each interval of $X$ (see Proposition 12 and Corollary 7).

Apart from that, we define the pseudo-inverse of a cdf as the infimum of a certain set. However, there is no guarantee that the infimum of each subset exists. That is the reason we restrict the definition to those points such that the infimum in the definition makes sense (see Definition 16), which always happens, for example, when we work with a compact LOTS. To overcome this problem, a further research line is to extend the cdf to the Dedekind-MacNeille completion where is does make sense to define pseudo-inverse without that problem (see [19]). Finally, we have shown that the pseudo-inverse of a cdf let us calculate integrals with respect to the probability measure we use to define the cdf (see Remark 5) and, also, let us generate samples of a distribution given by the probability measure by following the classical procedure (see Section 1) when we work in a compact separable LOTS (see Remark 6).

Please note that one of the main properties of a cdf is that it fully determines its probability measure, so a very interesting research line is to look for conditions to get this result in this new context. Indeed, in [22], the authors will give conditions to ensure that a cdf and its probability measure are univocally determined in a LOTS.

Author Contributions: These authors have contributed equally to the development of this theory and its writing.

Funding: This research was funded by Ministerio Español de Ciencia, Innovación y Universidades and FEDER grant number PGC2018-101555-B-I00, MINECO/FEDER grant number MTM2015-64373-P, UAL-FEDER grant number UAL18-FQM-B038-A and CDTIME.

Conflicts of Interest: The authors declare no conflict of interest.

\section{Abbreviations}

The following abbreviations are used in this manuscript:

LOTS Linearly ordered topological space

cdf Cumulative distribution function

\section{References}

1. Durante, F.; Sempi, C. Principles of Copula Theory; Taylor \& Francis Group: New York, NY, USA, 2015; ISBN 9780429066399.

2. Lowen, R. On the Existence of Natural Non-Topological, Fuzzy Topological Spaces; Heldermann: Berlin, Germany, 1985; ISBN 9783885382119.

3. Bronevich, A.G.; Rozenberg, I.N. Ranking probability measures by inclusion indices in the case of unknown utility function. Fuzzy Optim. Decis. Mak. 2014, 13, 49-71. [CrossRef]

4. Li, S.G. Fuzzy intervals. Appl. Math. Lett. 2001, 14, 737-740. [CrossRef]

5. Onasanya, B.O.; Hoskova-Mayerova, S. Some topological and algebraic properties of $\alpha$-level subsets topology of a fuzzy subset. An. St. Univ. Ovidius Constanta 2018, 26, 213-228. [CrossRef]

6. Menger, K. Statistical metrics. Proc. Natl. Acad. Sci. USA 1942, 28, 535-537. [CrossRef] [PubMed]

7. Šerstnev, A.N. Random normed spaces: Problems of completeness. Kazan Gos. Univ. Ucen. Zap. 1962, 122, 3-20.

8. Lafuerza-Guillén, B.; Harikrishnan, P. Probabilistic Normed Spaces; Imperial College Press: London, UK, 2014. 
9. Pourmoslemi, A.; Salimi, M. Probabilistic n-normed spaces, Dn-compact sets and Dn-bounded sets. Chaos Solitons Fractals 2009, 42, 2729-2734. [CrossRef]

10. Alimohammady, M.; Pourmoslemi, A.R. $\tau$-product of D-compact sets in probabilistic normed spaces. Int. J. Comput. Appl. Math. 2007, 2, 59-65.

11. Suck, R. A qualitative characterization of the exponential distribution. J. Math. Psychol. 1998, 42, $418-431$. [CrossRef] [PubMed]

12. Halmos, P.R. Measure Theory; Springer: New York, NY, USA, 1974; ISBN 0-387-90088-8.

13. Roman, S. Lattices and Ordered Sets; Springer: Irvine, CA, USA, 2008; ISBN 978-0-0387-78900-2.

14. Dushnik, D.; Miller, E.W. Partially ordered sets. Am. J. Math. 1941, 63, 600-610. [CrossRef]

15. Lutzer, D.J. On generalized ordered spaces. Diss. Math. 1971, 89, 42.

16. Steen, L.A.; Seedbach, J.A. Counterexamples in Topology; Springer: New York, NY, USA, 1978; ISBN 978-0-486-68735-3.

17. Schröder, B. Ordered Sets: An Introduction; Springer: Boston, MA, USA, 2003; ISBN 978-3-319-29786-6.

18. Chung, K.L. A Course in Probability Theory; Academic Press: San Diego, CA, USA, 2001; ISBN 0-12-174151-6.

19. Gálvez-Rodríguez, J.F.; Sánchez-Granero, M.A. The distribution function of a probability measure on the Dedekind-MacNeille completion. Topol. Appl. 2019, accepted for publication.

20. Bauer, H. Probability Theory and Elements of Measure Theory; Academic Press: London, UK, 1981; ISBN 3-11-013935-9.

21. Schervish, M.J. Theory of Statistics; Springer: Pittsburgh, PA, USA, 1995; ISBN 978-1-4612-8708-7.

22. Gálvez-Rodríguez, J.F.; Sánchez-Granero, M.A. Equivalence between Distribution Functions and Probability Measures on a LOTS. 2019, submitted for publication.

(C) 2019 by the authors. Licensee MDPI, Basel, Switzerland. This article is an open access article distributed under the terms and conditions of the Creative Commons Attribution (CC BY) license (http:/ / creativecommons.org/licenses/by/4.0/). 